\title{
Right Atrial Thrombus Mimicking Myxoma
}

\author{
Lalita Nemani ${ }^{1}$ Satish Killi ${ }^{1}$ \\ ${ }^{1}$ Department of Cardiology, Nizam's Institute of Medical Sciences, \\ Hyderabad, Telangana, India
}

Indian J Cardiovasc Dis Women-WINCARS 2017;2:86-90

\begin{abstract}
Address for correspondence Lalita Nemani, DM, Department of Cardiology, Nizam's Institute of Medical Sciences, Hyderabad, TS 500082, India (e-mail: drlalita775@gmail.com).
\end{abstract}

\begin{abstract}
Keywords

- deep vein thrombus

- myxoma

- protein C deficiency

- pulmonary embolism
\end{abstract}

Right atrial mass could be a tumor, thrombus, or vegetation, and it often poses a diagnostic dilemma. Accurate diagnosis is crucial to planning the correct management strategy. However, despite the advanced and sophisticated diagnostic modalities available, differentiating intracardiac masses could still be challenging. Clinical presentation leads to the appropriate conduit of investigations, and histopathology is confirmatory. When the diagnostic dilemma persists even after all efforts, clinical scenario should be strongly reconsidered, especially in unique clinical settings before concluding a diagnosis. In this article, the authors describe the case of a young woman with history of peripheral arterial thrombosis, who underwent surgical excision of a right atrial mass diagnosed as right atrial myxoma. She later presented with massive pulmonary embolism and deep vein thrombosis of the lower limbs. In view of her extensive thrombotic history, her entire case was reviewed including the histopathology slides, and the diagnosis of right atrial thrombus was considered and confirmed. On further workup for thrombotic state, she was found to have protein C deficiency.

\section{Introduction}

Right atrial (RA) myxoma is a rare entity, and RA thrombus is still rarer. Tricuspid valve vegetation can mimic an RA mass and is seen more frequently than either a tumor or a thrombus. RA thrombus is a rare manifestation of severe thromboembolic disease. In situ RA thrombi are usually immobile whereas secondary RA thrombus is mobile. Mobile RA thrombus usually coexists with massive pulmonary embolism (PE). ${ }^{1}$ Protein C deficiency is a hematologic abnormality with propensity to thromboembolic manifestations.

\section{Case Report}

A 32-year-old woman-nonhypertensive, nondiabetic, nonasthmatic-presented with shortness of breath class III-IV and swelling of both the lower limbs for the previous 10 days. The patient had history of acute left upper limb ischemia 2 years back for which she was admitted. Electrocardiogram (ECG) and echocardiogram were normal at that time. Diagnostic angiogram showed normal coronaries and left axillary artery thrombus with total cutoff for which she received catheter-guided thrombolysis with streptokinase. The patient was discharged in stable condition with pulses

Dol https://doi.org/ 10.1055/s-0038-1622964. restored in the left upper limb with advice for workup for prothrombotic state. She was admitted 1 month back at an outside hospital with shortness of breath class II and fever of 15 days. On evaluation, she was found to have an RA mass that was presumed to be RA myxoma after a transthoracic and transesophageal echocardiography, and she underwent surgical excision of the mass. The discharge summary from the cardiothoracic surgeon mentioned the RA mass as a mobile pedunculated mass with the stalk attached to the inferior vena cava-right atrium (IVC-RA) junction, occluding the tricuspid valve without much infiltration and was removed successfully. Histopathology showed a well-circumscribed mass, very focally covered by flat/cuboidal cells on the surface. Large areas of coagulative necrosis were present. There was tiny volume of viable tumor consisting of few stellate cells arranged in the cord, intimately admixed with slit-like blood vessels with areas of fresh hemorrhages and inflammatory cells. It was reported as cardiac myxoma with extensive infarction and necrosis.

Postoperatively, the patient continued to have breathlessness of class II and presented to the authors' institute with the present complaints. At presentation, she was hemodynamically stable, and clinical examination showed clear lungs with raised jugular venous pressure (JVP). Cardiovascular
License terms

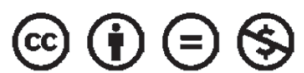




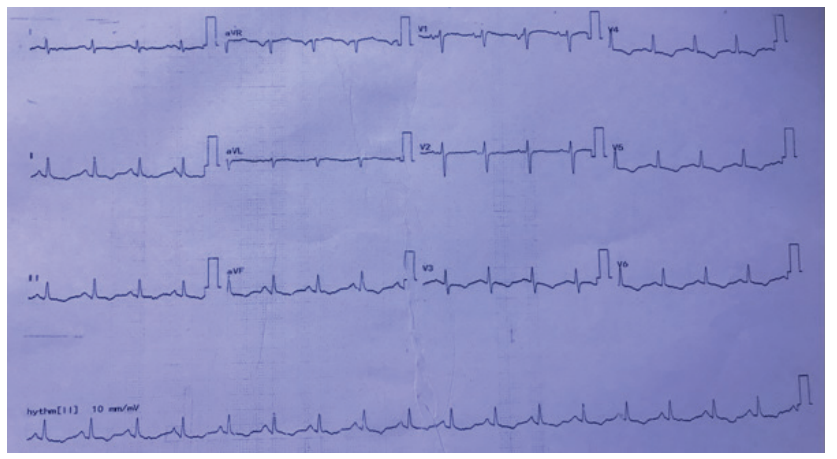

Fig. 1 ECG showing sinus tachycardia with T-wave inversion in leads II, III, aVF, and $\mathrm{V}_{3}$ to $\mathrm{V}_{6}$.

examination showed moderate pulmonary arterial hypertension (PAH). ECG showed T-wave inversion in leads II, III, $\mathrm{aVF}$, and $\mathrm{V}_{3}$ to $\mathrm{V}_{6}($ - Fig. 1). Chest radiograph showed RA enlargement with dilated pulmonary artery $(-$ Fig. 2$)$. Transthoracic two-dimensional (2D) echocardiogram demonstrated dilated RA, right ventricle (RV) with mild right ventricular (RV) dysfunction, and normal left ventricular (LV) systolic function. There was severe tricuspid regurgitation (TR) and moderate PAH with RVSP of $50 \mathrm{~mm} \mathrm{Hg}$. There was no mitral regurgitation/aortic regurgitation (MR/AR), pericardial effusion, vegetation, or clots. Venous Doppler of both lower limbs showed acute thrombus involving the left common and superficial femoral veins. Computed tomography (CT) pulmonary angiography showed saddle thrombus at the level of pulmonary trunk bifurcation extending into right main pulmonary artery ( - Fig. 3 ). The patient was treated with heparin infusion followed by oral anticoagulants and supportive therapy. Thrombotic profile analysis was done. Anticardiolipin antibodies showed negative immunoglobulin G $(\operatorname{IgG})$, but immunoglobulin M (IgM) was positive. Antinuclear antibody (ANA) was 2+ with homogenous pattern. Serum homocysteine level was $26.4 \mu \mathrm{mol} / \mathrm{L}$. In view of history of arterial thrombus, RA mass attached to IVC-RA junction (which is not a common site for myxoma), histopathology-not classic

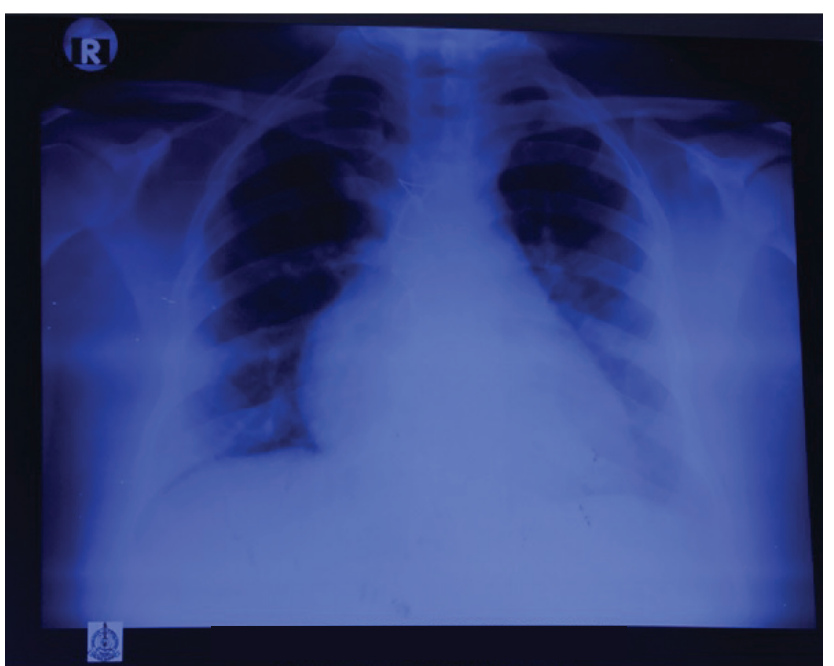

Fig. 2 PA view of chest radiograph showing cardiomegaly with right atrial enlargement with dilated pulmonary artery. description of myxoma-and now presenting with saddle pulmonary artery embolus with hyperhomocysteinemia and abnormal collagen profile, the authors thought of RA mass as thrombus rather than myxoma. Histopathology slides were reviewed at the institute, and the pathologist unanimously opined them as thrombus. The patient was discharged on oral anticoagulants with advice of further workup for thrombotic state. She lost regular follow-up and was noncompliant with medication.

One year later, the patient again presented with shortness of breath of class III and bilateral pedal edema of 3 months duration. Clinically, there were features of right-sided heart failure. On evaluation, ECG was suggestive of RV strain (-Fig. 4). Two-dimensional (2D) echo showed dilated RA, RV with RV dysfunction, severe TR with severe PAH (RVSP = $96 \mathrm{~mm} \mathrm{Hg}$ ). Chest X-ray showed the same features as before (-Fig. 5). Repeated CT pulmonary angiogram was done as there was exacerbation of symptoms, which showed chronic pulmonary thromboembolism with recanalization in right lower lobe pulmonary arteries and marked attenuation of left lower lobe pulmonary artery likely sequelae of chronic thrombosis (-Fig. 6). Complete blood picture and renal parameters were normal. ANA and anticardiolipin $(\mathrm{aCL})$ antibodies were negative, homocysteine levels were normal, and protein S was normal. However, protein C was very low $29 \%$ (normal 70-130\%). Anti-PAH drug therapy was started, and oral anticoagulation tapered to ideal international normalized ratio (INR) was continued.

\section{Discussion}

Myxomas are the most common intracardiac tumors. Atrial myxoma accounts for 35 to $50 \%$ of primary cardiac tumors of which only $20 \%$ arise from the RA. ${ }^{2,3,4} \mathrm{~A}$ typical RA myxoma is solitary pedunculated arising from the fossa ovalis or base of the interatrial septum. Occasionally they arise from the atrial wall, appendage, and very rarely tricuspid and eustachian valve. The diagnosis is usually made by $2 \mathrm{D}$ echocardiography; however, transesophageal echocardiography is superior for delineation of size, exact location, morphology, and the point of attachment.

The classic echocardiographic features to differentiate myxoma from atrial thrombus are that the thrombi are irregular, laminated, and immobile with a broad base attached to the posterior atrial wall. ${ }^{5}$ However, this description holds good only for in situ atrial thrombi and not for secondary atrial thrombi. Secondary atrial thrombi are usually from the peripheral veins and are hence mobile. These are also referred to as "emboli in transit" as they on their way to the pulmonary arteries. ${ }^{6}$ They are described as spherical, coiled, grapelike, ovoid, worm-like, or serpiginous masses moving within the RA. When large, they may prolapse through the tricuspid valve into the RV. Often, these masses appear free floating with no attachment site. Such mobile thrombi are very often mistaken for RA myxoma.

In this patient, features favoring RA thrombi are history of repeated thromboembolic episodes, initial normal echocardiogram (less likely in case of myxoma), and abnormal 


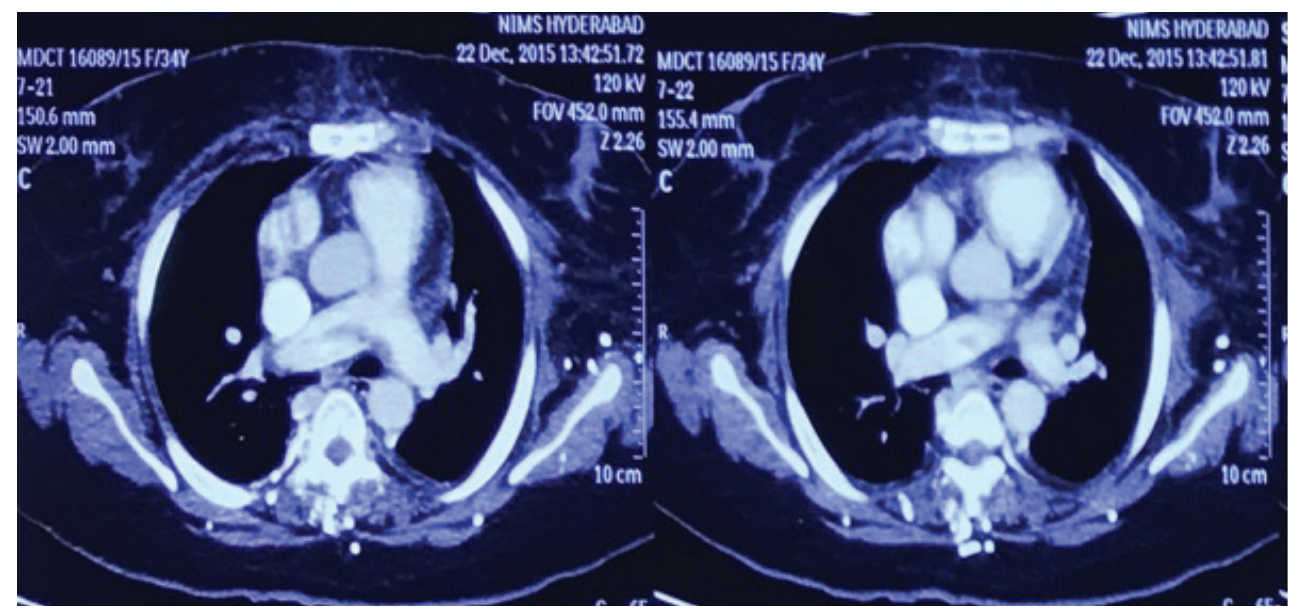

Fig. 3 CT pulmonary angiogram showing saddle thrombus with extension into right pulmonary artery.

attachment of the mass (to the IVC-RA junction). Large stellate cells with vacuolated cytoplasm in the myxoid background on histopathologic examination is pathognomonic of myxoma. ${ }^{7}$ However, the classic description was missing in the initial histologic report commenting as probable myxoma.

Intracardiac thrombus could be the manifestation of primary cardiac abnormality or hematologic disorder or rheumatologic disease. It is common in those with indwelling catheter in the RA such as those on hemodialysis. Echocardiographic features suggestive of primary cardiac pathology such as hypokinetic chambers, valvular abnormality, and spontaneous echo contrast were absent in this case, excluding cardiac source of thromboembolism.

Cardiac thrombus in the absence of primary cardiac disease is less likely, but it can occur in the setting of hypercoagulable state. The risk of intracardiac thrombosis is increased in patients who have systemic lupus erythematosus (SLE) and who either show positive test results for lupus anticoagulant activity or have medium or high levels of anticardiolipin antibodies. ${ }^{8}$ A retrospective study of 637 patients $^{3}$ found that venous thrombosis with PE was more frequent in persons who had lupus anticoagulant activity, whereas coronary, cerebrovascular, and peripheral arterial events were more likely in individuals who had elevated levels of IgG or IgM anticardiolipin antibodies. ${ }^{8}$

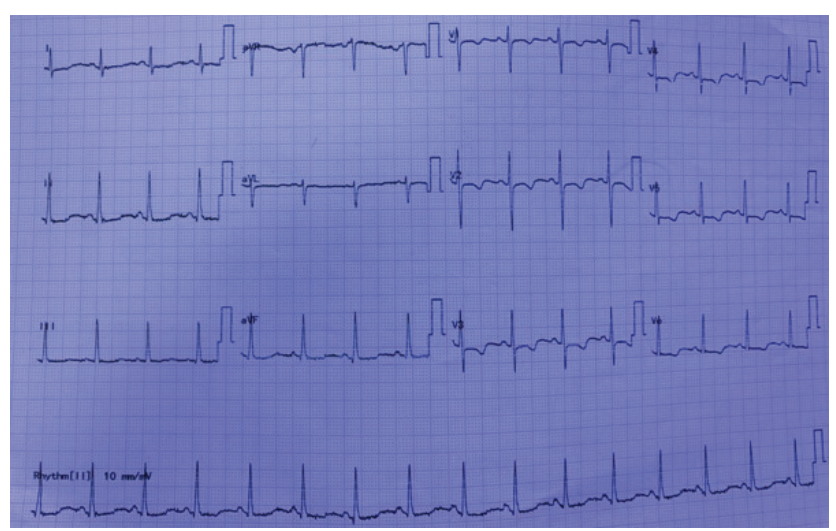

Fig. 4 ECG showing RVH with strain pattern.
Collage profile showed some abnormality, but the rheumatologist opined that it was not specific for any rheumatologic disorder

The most likely cause for thrombus formation in this patient was protein C deficiency that was $29 \%$ against a normal of 70 to $130 \% .{ }^{9}$ Protein C has both anticoagulant and profibrinolytic properties. It inactivates factors Va and VIIIa, and protein $\mathrm{S}$ is a cofactor for these actions. Hence proteins $\mathrm{C}$ and $\mathrm{S}$ deficiency predisposes to the formation of arterial and venous thrombi. These patients usually develop venous thrombotic complications between the ages of 15 and 40 years with a high incidence of deep venous thrombosis (DVT) and PE. Thrombosis can also occur in the cerebral, retinal, mesenteric, and renal veins and the inferior vena cava. Presentation as right-side cardiac thrombus is the result of fragmentation of the thrombus and subsequent pulmonary embolization.

Many other rare mimics of RA myxoma have been described in literature. Khanna et al reported mimicker of RA mass due to sinus of Valsalva's aneurysm. An asymptomatic 55-year-old man of unruptured sinus of Valsalva's aneurysm of noncoronary cusp was on medical follow-up.

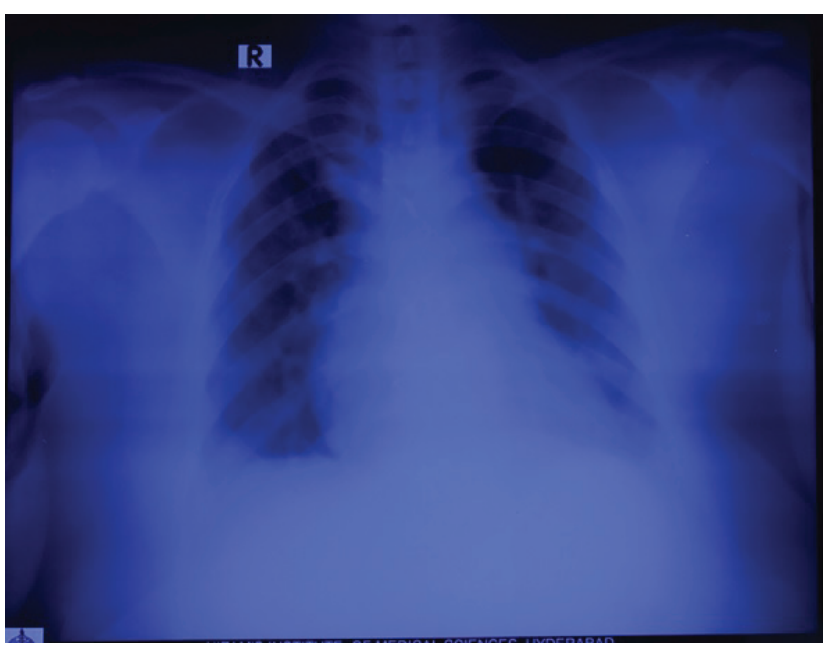

Fig. 5 Chest radiograph showing right atrial and pulmonary arterial dilatation. 


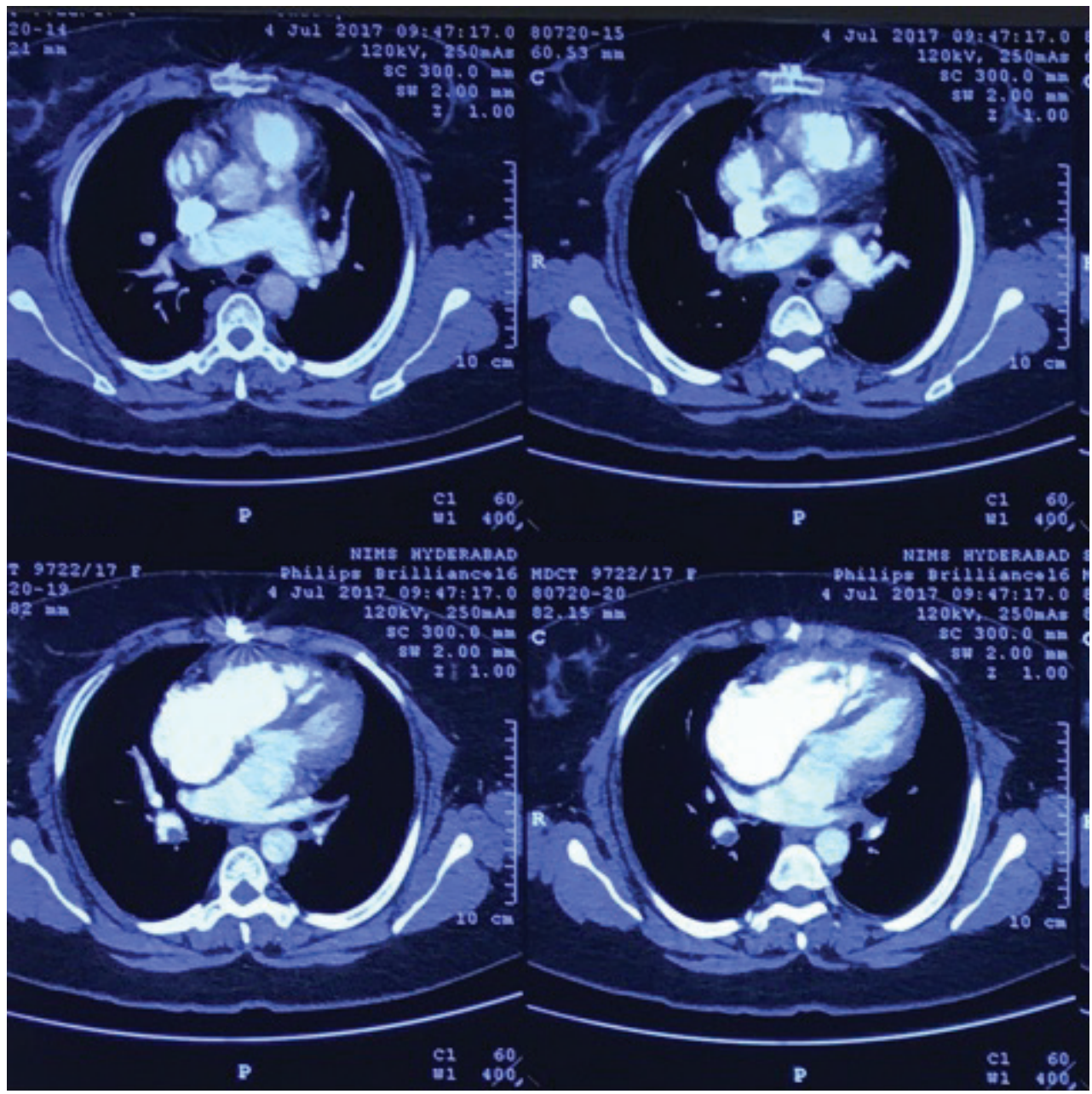

Fig. 6 Second CTPA showing evidence of old thrombus with partial recanalization in the MPA and RPA.

At 2-year follow-up, there was thrombus formation in the aneurysm, mimicking RA tumor on 2D transthoracic echocardiography. Cardiac CT showed filling defect in the aneurysm suggestive of thrombus. ${ }^{10}$ Garg et al report the case of a 53-year-old woman with RA mass mimicking myxoma, which the histopathologic evaluation revealed to be Rosai-Dorfman disease (sinus histiocytosis with massive lymphadenopathy) of the RA. ${ }^{11}$ Garg et al and Li et al presented a case of RA mass due to paraganglioma that is pedunculated like in this case. ${ }^{12}$ Li et al demonstrated pedunculated mass mimicking RA myxoma, tuned out to be a thrombus. ${ }^{13}$ Salani et al presented the case report of a 19-year-old patient with chronic kidney disease due to chronic glomerulonephritis, in hemodialysis (HD) by central catheter, with the incidental finding of a mass of $28 \times 16 \mathrm{~mm}$ in the RA. The diagnosis of thrombus, infective endocarditis, or myxoma was considered. After 180 days of anticoagulation, there was significant reduction in mass. ${ }^{14}$

RA thrombus is seen in $4 \%$ of patients diagnosed as PE and is associated with increased mortality. ${ }^{15}$ Treatment options are controversial as there are limited data for comparison. Initial treatment with heparin and follow-up with oral anticoagulation form the primary cornerstone of treatment. Large thrombi require surgical excision as thrombectomy may not suffice. The mortality rate associated with no therapy, anticoagulation therapy, embolectomy, and thrombolysis were $100 \%, 29 \%, 24 \%$, and $11 \%$, respectively. ${ }^{16}$ Surgery may be insufficient in case of multiple thrombi in which thrombolysis is favored despite the risk of migration of thrombi. Low-dose and prolonged fibrinolysis is recommended to reduce the risk of thrombus migration.

\section{Conclusion}

The diagnostic dilemma of an RA mass could persist despite all investigations and histopathology unless clinical scenario is taken into consideration. RA thrombus should be considered in the close differential diagnosis of RA mass, especially in the clinical context of multiple thromboembolic events. Treatment should be individualized owing to lack of evidence. Patients should be evaluated for rheumatologic disorders and hypercoagulable state. All patients should be evaluated for proteins $C$ and $S$ deficiency in the presence of unprovoked DVT and PE. 


\section{References}

1 Kane GR, Deshpande S, Mallakmir S, Laddha M. Atrial thrombus mimicking atrial myxoma in protein $C$ deficiency patient. Int Med J 2014;1(8):455-458

2 Lenihan DJ, Yusuf SW. Tumors affecting the cardiovascular system. In: Bonow RO, Mann DL, Zipes DP, Libby P, Braunwald E, eds. Braunwald's Heart Disease: A Textbook of Cardiovascular Medicine. 10th ed. Philadelphia, PA: Elsevier Saunders; 2015:chap 85

3 McKenna WJ, Elliott P. Diseases of the myocardium and endocardium. In: Goldman L, Schafer AI, eds. Goldman's Cecil Medicine. 25th ed. Philadelphia, PA: Elsevier Saunders; 2016:chap 60

4 Mitchell MC, Boitnott JK, Kaufman S, Cameron JL, Maddrey WC. Budd-Chiari syndrome: etiology, diagnosis and management. Medicine (Baltimore) 1982;61(4):199-218

5 Pores IH, Abel RM, Gray L, Jacobs GP. Giant right atrial myxoma with rheumatic mitral valve disease. Angiology 1984;35(5):313-319

6 Lazar L, Dave R, Tabibiazar R. Dilemma of right atrial thrombi, to dissolve or to extract. Proceedings of UCLA Healthcare 2012;16:1

7 Zheng JJ, Geng XG, Wang HC, Yan Y, Wang HY. Clinical and histopathological analysis of 66 cases with cardiac myxoma. Asian Pac J Cancer Prev 2013;14(3):1743-1746

8 Soltész P, Veres K, Lakos G, Kiss E, Muszbek L, Szegedi G. Evaluation of clinical and laboratory features of antiphospholipid syndrome: a retrospective study of 637 patients. Lupus 2003;12(4):302-307

9 Ercan E, Tengiz I, Sekuri C, et al. Cardiac thrombi in a patient with protein-C and $\mathrm{S}$ deficiencies: a case report. Thromb J 2004;2(1):2

10 Khanna R, Shah P, Dey M, Goel PK, Goyal P, Pandey S. Unruptured sinus of Valsalva aneurysm mimicking as right atrial tumor. Echocardiography 2017;34(7):1107-1109

11 Khanna SN, Iyer KS, Paul M, Radhakrishnan S, Rathi AK. Rosai-Dorfman disease of right atrium mimicking myxoma. Ann Thorac Surg 2017;103(5):e443-e445

12 Garg A, Mishra D, Bansal M, Maharia HR, Goyal V. Right atrial paraganglioma: an extremely rare primary cardiac neoplasm mimicking myxoma. J Cardiovasc Ultrasound 2016;24(4): 334-336

13 Li X, Chen L, Duan X, Wang X. A rare case of a pedicled mobile thrombus in right atrium. Heart Surg Forum 2016;19(6):E269-E271

14 Salani TG, Borges CM, Urbini CS, et al. Patient in chronic hemodialysis with right atrial mass: thrombus, fungal endocarditis or atrial myxoma? J Bras Nefrol 2016;38(4):462-465

15 Lobo A, Lewis JF, Conti CR. Intracardiac masses detected by echocardiography: case presentations and review of the literature. Clin Cardiol 2000;23(9):702-708

16 Rose PS, Punjabi NM, Pearse DB. Treatment of right heart thromboemboli. Chest 2002;121(3):806-814 